\title{
O uso de politetrafluoroetileno expandido em tireoplastia tipo I
}

\section{The use of expanded polytetrafiuoroethylen by type I thyroplasty}

\author{
Francisco Veríssimo de Mello-Filho', \\ Lílian Ricz², Leonardo de S. Kruschewsky ${ }^{3}$
}

Palavras-chave: politetrafluoroetileno expandido, gore-tex, paralisia de corda vocal, tireoplastia, politef.

Key words: vocal cord paralysis, thyroplasty, gore-tex, polytef, expanded polytetrafluoroethylene.

\section{Resumo / Summary}

\section{A} paralisia unilateral de prega vocal (PUPV) em abdução é uma alteração que produz grande desconforto ao paciente, principalmente pelos distúrbios fonatórios causados. Atualmente dispomos de uma variedade de métodos para seu tratamento. Objetivo: Verificar a eficiência, vantagens e desvantagens do uso da fita de politetrafluoretileno expandido (PTFE-e), na medialização de prega vocal (PV) paralisada, em abdução. Forma de Estudo: Coorte Longitudinal. Material e Métodos: Foram estudados quatro pacientes com diagnóstico clínico-laringoscópico de disfonia por PPVU, em abdução há pelo menos um ano. Os pacientes foram submetidos a avaliação fonoaudiológica e a tireoplastia tipo I para medialização da PV com fita de PTFE-e. Durante o seguimento pós-operatório procedeu-se videolaringoscopia, tomografia computadorizada e fonoterapia. Resultados: Todos os pacientes estão satisfeitos com a qualidade vocal, apresentam medialização estável da PV com a fonocirurgia e bons resultados à avaliação fonoaudiológica. Um paciente apresentou extrusão intra-operatória da fita de PTFE-e, sendo feito um segundo procedimento, sem complicações, seis meses depois. Conclusão: Na opinião dos autores a fita de PTFE-e se presta com eficiência ao tratamento da PUPV, sem apresentar riscos importantes.

\begin{abstract}
$T$ he Unilateral Vocal Fold Paralysis (UVFP), abducted, is a medical pathology that gives a lot of discomfort to the patient especially because of the speech impairment. Nowadays it is offered a variety of scientific methods for its treatment. Aim: To study the efficacy, advantages and disadvantages in using expanded Polytetrafluoroethylene (e-PTFE)'s band to medialize a paralyzed and abducted vocal fold. Study Design: Longitudinal Cohort. Material and Methods: Four patients with medical-laryngoscope diagnosis of disphonia by UVFP, abducted for at least one year. They were submitted to speech pathology evaluation and so, to e-PTFE type one thyroplasty medialization and followed by videolaryngoscopy, computed tomography and speech pathology therapy. Results: All four patients were satisfied with the thyroplasty results, videolaryngoscopy was used to document the vocal fold medialized and its function was studied by speech pathologist. One patient had e-PTFE band extrusion during surgery, being submitted of another procedure six months after without complications. Conclusion: PTFE band is an efficient treatment for UVFP abducted without important risks.
\end{abstract}

\footnotetext{
${ }^{1}$ Professor Doutor da Disciplina de Cirurgia de Cabeça e Pescoço do Departamento de Oftalmologia, Otorrinolaringologia e Cirurgia de Cabeça e Pescoço do Hospital das Clínicas da Faculdade de Medicina da Universidade de São Paulo, Campus Ribeirão Preto. ${ }^{2}$ Fonoaudióloga e Coordenadora do Serviço de Fonoaudiologia do Hospital das Clínicas da Faculdade de Medicina da Universidade de São Paulo, Campus Ribeirão Preto. ${ }^{3}$ Médico Contratado da Disciplina de Cirurgia de Cabeça e Pescoço do Departamento de Oftalmologia, Otorrinolaringologia e Cirurgia de Cabeça e Pescoço do Hospital das Clínicas da Faculdade de Medicina da Universidade de São Paulo, Campus Ribeirão Preto. Endereço para Correspondência: Francisco Veríssimo de Mello-Filho - Rua Salvador Delloiagono, 70 Jardim Canada Ribeirão Preto SP Tel/Fax (0xx16) 623-6169 / 602-2353 - E-mail:fdmfilho@yahoo.com

Artigo recebido em 18 de novembro de 2002. Artigo aceito em 08 de agosto de 2003.
} 


\section{INTRODUÇÃO E OBJETIVO}

Paralisia unilateral de prega vocal (PUPV) em abdução é um fator importante de disfonia e de manifestação clínica variada, na dependência do prejuízo que traga ao fechamento glótico. Quanto mais abducente for a posição da prega vocal (PV) paralisada, maior a incompetência glótica e maiores são as conseqüências negativas para a fonação do paciente ${ }^{1,2}$.

A PUPV pode ser de origem central ou periférica e pode ter várias etiologias ${ }^{2,3}$. As centrais podem ser decorrentes de tumores, acidentes vasculares e traumáticos. As periféricas ocorrem principalmente por iatrogenias, decorrente de cirúrgicas cérvico-torácicas, neoplasias cérvico-torácicas com envolvimento do nervo laríngeo recorrente e/ ou do nervo vago, traumáticas, e idiopáticas.

A fonocirurgia, com técnicas que promovem a medialização da PV paralisada abducente, tem demonstrado grande avanço nos últimos anos. Atualmente dispomos de uma quantidade razoável de opções cirúrgicas e de materiais que possibilitam a medialização da PV ${ }^{413}$. Entre as opções cirúrgicas podemos utilizar a via endoscópica ${ }^{14}$, com injeção de diversos tipos de materiais na PV 2,3,6-10 ou a via cervical aberta, com a execução de tireoplastias, modificações do esqueleto cartilaginoso da laringe e rotação da aritenoide ${ }^{4,5,15-17}$.

Entre estas, a tireoplastia tipo I tem mostrado ser uma das melhores opções para a medialização da PV15,18,19, entretanto ainda é discutível qual seria o melhor material utilizado como implante entre a cartilagem tireóide e os tecidos moles da região glótica. É possível o uso de material sintético como silicone, teflon, e a fita de PTFE- $e^{4-9,14,18-20}$, ou ainda material biológico como cartilagem, fascia muscular ou gordural-4,10-14,21,22 com o mesmo propósito, sendo que os dois últimos, junto com o teflon, são utilizados para medialização por injeção.

Com o objetivo de se verificar a eficiência, vantagens e desvantagens do uso de fita de PTFE-e na medialização de PV paralisada, testamos o seu uso associado à técnica de tireoplastia tipo I preconizada por Isshiki, no tratamento de quatro pacientes com PUPV em abdução.

\section{MATERIAL E MÉTODO}

Entre julho de 2001 e abril de 2002, no HC-FMRPUSP, quatro pacientes com PUPV foram tratados por tireoplastia tipo I e medialização de PV abducente com o uso de fita de PTFE-e $(6 \mathrm{~mm}$ de diâmetro por $50 \mathrm{~cm}$ de comprimento).

Adotamos como critérios de inclusão neste estudo os seguintes itens:

a) Diagnóstico de PUPV abducente por um período superior a um ano.

b) Não ter sido submetido previamente a outro tratamento para medialização da PV paralisada. c) Fonoterapia pré-operatória sem sucesso para compensação vocal adequada.

d) Consentimento pós-informação do paciente em se submeter à cirurgia proposta, sob anestesia local.

Todos os pacientes foram submetidos à avaliação clínica, laboratorial, fonoaudiológica e a videolaringoscopia pré-operatória.

Após a cirurgia os pacientes foram submetidos a fonoterapia, acompanhamento videolaringoscopico a cada dois meses e estudo de imagem com tomografia computadorizada até os seis primeiros meses.

Para análise da qualidade vocal, gravou-se a voz dos pacientes em fitas cassetes de marcas comerciais, em gravador Sony ${ }^{\circledR}$, com microfone de lapela acoplado ao gravador, situado a $10 \mathrm{~cm}$ dos falantes, em cabina acústica. Solicitou-se ao paciente a emissão prolongada das vogais "a", "i", u", contagem de 1 a 30 e o canto "Parabéns a Você". Foram instruídos a produzir a voz e a fala na intensidade e freqüência vocais habituais.

As amostras foram analisadas por fonoaudiólogos com conhecimento em voz. Efetuou-se a análise perceptiva auditiva utilizando a escala "GRBAS", validada por Hirano, em 1981, que caracteriza o grau de disfonia (G-grade), avaliando entre 0 e 3 , a rouquidão (R-roghness), a soprosidade (B-breathiness), a astenia (A-asthenicity) e a tensão (S-strain), no pré e pós-medialização da prega vocal paralisada com o uso de fita de PTFE-e. Aplicou-se a escala "GRBAS", com a graduação obedecendo ao critério de ausência da disfonia (0), alteração discreta (1), alteração moderada (2) e alteração severa (3).

\section{Pacientes}

Paciente 1, R. C. M. S., 16 anos, sexo feminino, portadora de atresia tricúspide e submetida a cirurgia cardíaca para anastomose átrio-pulmonar esquerda. Apresentou no pós-operatório PUPV esquerda.

Paciente 2, J.R.L., 22 anos, sexo masculino, vítima de ferimento por arma branca em região cervical esquerda evoluindo com PUPV esquerda.

Paciente 3, J.R., 51 anos, sexo masculino, portador de tumor mesenquimal, paraganglioma de artéria carótida esquerda, operado em novembro de 2000 e evoluindo com PUPV esquerda.

Paciente4, L.A.C., 48 anos, sexo masculino portador de paraganglioma de tronco de nervo vago localizado em sua emergência do forame jugular direito, operado em fevereiro de 2001 sendo seccionado o nervo vago e retirado o segmento tumoral, evoluindo com PUPV direita.

\section{Técnica cirúrgica}

No preparo pré-cirúrgico foi utilizada sedação venosa leve pelo anestesiologista e anestesia tópica com spray de lidocaina $10 \%$ borrifada em mucosa nasal e de orofaringe. 
Na região cervical foi injetado lidocaina $2 \%$ com adrenalina $1 / 200.000 \mathrm{U}$ em topografia da borda inferior da cartilagem tireóide do lado da PUPV. A seguir, o nasolaringofibroscópio era passado por via nasal e posicionado logo acima da laringe oferecendo boa visibilidade das PVs durante todo 0 procedimento.

A técnica utilizada foi a tireoplastia tipo I preconizada por Isshiki ${ }^{4,5,15}$. Após o descolamento do pericôndrio interno da cartilagem tireóide inicia-se a introdução da fita de PTFEe pela abertura em janela na cartilagem tireóide. De maneira contínua, é introduzida progressivamente a fita de PTFE-e, empurrando a PV para a posição mediana, sob visualização fornecida pela videolaringofibroscopia. Q uando a PV atinge a linha mediana, solicitamos ao paciente que emita sons para verificarmos a qualidade vocal. Neste momento, em conjunto com uma fonoaudióloga, passamos a modular a voz do paciente introduzindo mais anteriormente ou posteriormente a fita de PTFE-e até atingir uma fonação satisfatória e um posicionamento tridimensional adequado daPV.

\section{RESULTADOS}

O s resultados sonoros foram excelentes nos quatro pacientes. Não houve qualquer manifestação de sangramento ou dispnéia nos pacientes, enquanto internados, permitindo sua alta já no primeiro pós-operatório. Não ocorreu infecção da ferida cirúrgica, febre, reação granulomatosa ou rejeição biológica do PTFE-e durante todo o seguimento.

0 paciente 4 apresentou como complicação a ruptura acidental do seio piriforme durante a colocação da fita de PTFE-e na primeira cirurgia, que foi de pronto identificado sendo interrompido o procedimento. 0 paciente evoluiu bem, com tratamento clínico baseado em jejum via oral, hidratação venosa por três dias e antibioticoterapia seguido de introdução de dieta líquida, após prova do azul de metileno negativa, no quarto dia.

Seis meses depois o paciente foi novamente submetido ao procedimento de medialização da PV com PTFE-e sem apresentar qualquer problema e apresentando o mesmo grau de satisfação dos outros pacientes.

Os achados fonoaudiológicos e laringoscópicos são mostrados nas Tabelas 1 e 2 comparando os resultados pré e pós-operatórios.

As imagens da laringoscopia, com o paciente em fonação, produzindo adução máxima das PV, são mostradas no pré-operatória (Figura 1) e pós-operatória (Figura 2).

$\mathrm{Na}$ Figura 3 é mostrado o estudo de imagem da laringe, com tomografia computadorizada, seis meses após medialização da PV com PTFE-e.

\section{DISCUSSÃO}

Embora citado em um grande número de séries $2,8,19,23$ a PUPV com etiologia idiopática, em nossa pequena amostra, os quatro casos apresentavam evidentes fatores etiológicos.

Tabela 1. Descrição dos resultados obtidos com análise perceptiva auditiva nos pacientes tratados com medialização de prega vocal abducente com fita de Gore-Tex de acordo com a escala GRBAS.

\begin{tabular}{lcccccccc}
\hline GRBAS & \multicolumn{2}{c}{ PACIENTE 1 } & \multicolumn{2}{c}{ PACIENTE 2 } & \multicolumn{2}{c}{ PACIENTE 3 } & \multicolumn{2}{c}{ PACIENTE 4 } \\
& PRÉ-OP. & PÓS-OP. & PRÉ-OP. & PÓS-OP. & PRÉ-OP. & PÓS-OP. & PRÉ-OP. & PÓS-OP. \\
\hline $\begin{array}{l}\text { G GRAU DA DISFONIA } \\
\text { R }\end{array}$ & 2 & 0 & 3 & 1 & 2 & 1 & 3 & 1 \\
$\begin{array}{l}\text { ROUQUIDÃO } \\
\text { B }\end{array}$ & 2 & 0 & 3 & 1 & 2 & 1 & 3 & 1 \\
$\begin{array}{l}\text { SOPROSIDADE } \\
\text { A }\end{array}$ & 2 & 0 & 3 & 1 & 2 & 1 & 3 & 1 \\
$\begin{array}{l}\text { ASTENIA } \\
\text { S }\end{array}$ & 0 & 0 & 0 & 0 & 1 & 0 & 0 & 0 \\
TENSÃO & 0 & 0 & 0 & 0 & 0 & 0 & 0 & 0 \\
\hline
\end{tabular}

Tabela 2. Achados da laringoscopia descrevendo aspectos da prega vocal medializada, pré e pós-operatórios.

\begin{tabular}{|c|c|c|c|c|}
\hline & \multicolumn{2}{|c|}{ POSIÇÃO DA P. V. } & \multirow{2}{*}{$\begin{array}{l}\text { ONDA DE MUCOSA } \\
\text { PRÉ \& PÓS }\end{array}$} & \multirow{2}{*}{$\begin{array}{l}\text { ATROFIA DA P. V. } \\
\text { PRÉ \& PÓS }\end{array}$} \\
\hline & PRÉ & PóS & & \\
\hline PACIENTE 01 & ABDUÇÄO & ADUÇÄO & NORMAL & AUSENTE \\
\hline PACIENTE 02 & ABDUÇÃO & ADUÇÃO & NORMAL & PRESENTE \\
\hline PACIENTE 03 & ABDUÇÃO & ADUÇÃO & NORMAL & PRESENTE \\
\hline PACIENTE 04 & ABDUÇÃO & ADUÇÃO & NORMAL & AUSENTE \\
\hline
\end{tabular}




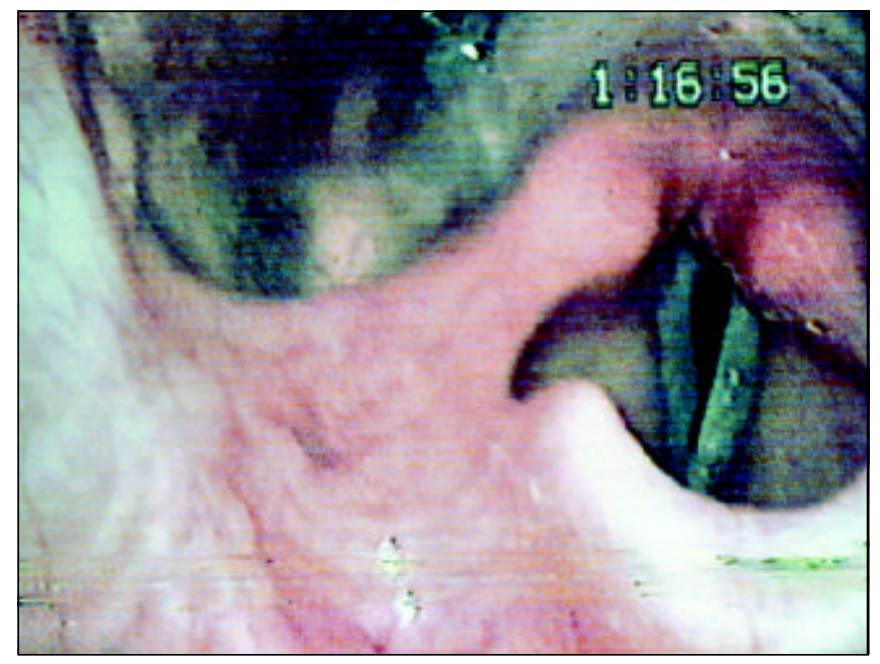

Figura 1. Laringoscopia pré-operatória, com paciente em adução máxima.

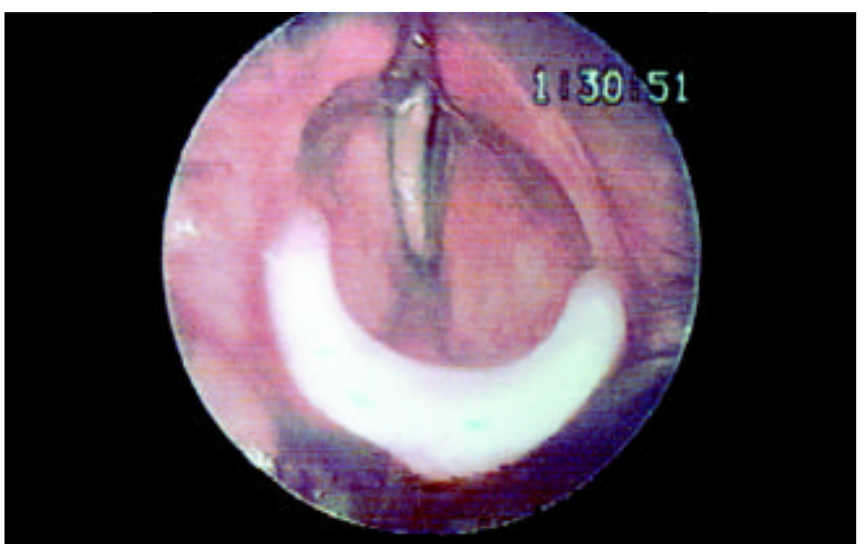

Figura 2. Laringoscopia pós-operatória, com fechamento glótico satisfatório.

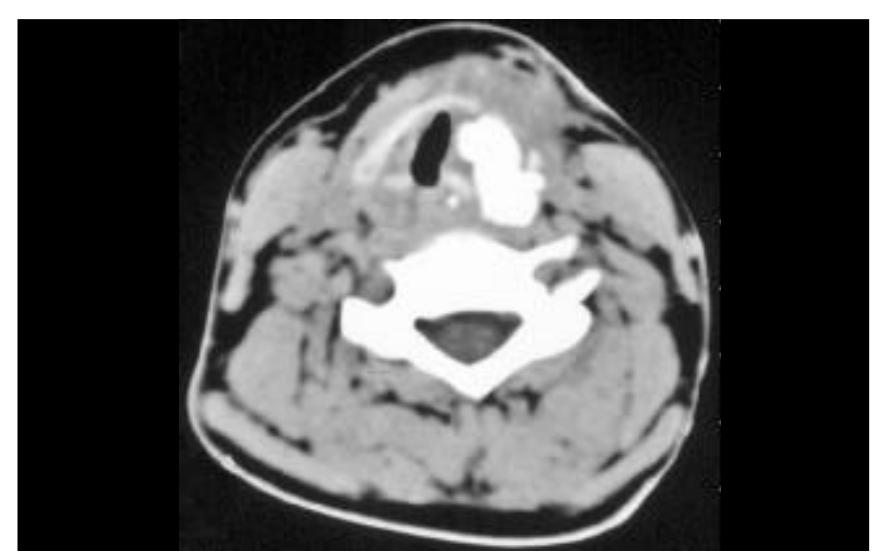

Figura 3. Imagem da tomografia computadorizada no pós-operatório, documentando o implante de Gore-Tex localizada no espaço paraglótico, medializando a prega vocal.
Chama a atenção a diversidade de etiologias para a PUPV, entretanto, nesse estudo, a maioria dos casos foi decorrente de procedimentos cirúrgicos.

Foi estabelecido um tempo de observação clínica de no mínimo um ano, para verificar a ausência de retorno expontâneo da mobilidade da PV ${ }^{19}$. Este prazo, entretanto, pode ser encurtado, especialmente em situações clínicas em que a PUPV esteja criando grande desconforto ou pela própria ansiedade do paciente. Nestes casos é recomendável fazer um estudo eletromiográfico da laringe, mas de qualquer forma devemos lembrar que a medialização da PV com fita de PTFE-e pode ser revertida a qualquer momento, para isso bastando remover o implante.

Os pacientes deste estudo não haviam sido submetidos a qualquer outro tipo de procedimento para medialização da PV, bem como nenhuma medida adicional foi acrescentada ao uso do PTFE-e. Este cuidado foi tomado para podermos analisar isoladamente apenas o resultado obtido com o PTFE-e. É possível associar outras medidas como injeção de substâncias na PV ou rotação da aritenóide para conseguir um bom ajuste vocal, entretanto estas medidas não se fizeram necessárias em nenhum dos casos.

Fonoterapia pré-operatória foi aplicada como tentativa de conseguir a compensação vocal, bem como para avaliação do grau de disfonia. No pós-operatório a fonoterapia colaborou na adaptação vocal e avaliação da qualidade vocal.

A predominância de paralisia de PV esquerda, encontrada em nossa série, também se repete em outras séries $^{23-25}$. É possível que tal fato ocorra apenas devido à peculiaridade anatômica do nervo laríngeo recorrente do lado esquerdo, entretanto não conhecemos nenhum trabalho que estabeleça esta comprovação científica, cabendo apenas seu registro.

O uso dos blocos de silicone ou de cartilagem para medialização da PV possui alguns inconvenientes. Eles necessitam ser moldados e manipulados no momento da cirurgia ${ }^{14,15}$ para chegar a uma forma ideal ou seja, uma medida capaz de entrar na janela da cartilagem tireóide e produzir completa medialização da PV, o que por vezes, torna-se trabalhoso estendendo o tempo cirúrgico.

O uso da fita de PTFE-e demonstrou ser de fácil manuseio especialmente pela característica de maleabilidade da fita, tornando o procedimento mais fácil e rápido ${ }^{19}$. A despeito de ser possível conseguir uma boa medialização da PV com o bloco sólido de silicone, muitas vezes é necessário também o alongamento, retificação de alguma deformidade e nivelamento da PV, o que geralmente não é possível com o bloco de silicone. A fita de PTFE-e possibilita o micro ajuste da PV, após colocar uma quantidade variável da fita no compartimento laríngeo e conseguir sua medialização, passamos ao ajuste fino do posicionamento anatômico das PVs, sob visão endoscópica e sonora com a emissão de sons pelo paciente ${ }^{15,18-21}$. Se ocorrer deformidade da PV, com a formação de alguma concavidade, convexidade 
ou desnivelamento, é possível introduzir ou extrair pequena quantidade da fita, na região anterior, média ou posterior da PV, de forma a buscar a sua simetria e alinhamento. É possível também buscar 0 alongamento da PV, colocando-se mais PTFE-e junto à comissura posterior, empurrando a cartilagem aritenóide para a região posterior, manobra praticamente utilizada em todos os pacientes; em um caso (caso 4), durante esta manobra, acabamos perfurando o seio piriforme com a saída da fita de PTFE-e pelo hipofaringe.

Em relação às substâncias injetáveis para a medialização da PV, podemos utilizar as biológicas como gordura, colágeno ou fascia, que possuem o inconveniente de serem absorvidas de maneira imprevisível $2,3,14,18,21,23,24$. As substâncias sintéticas injetáveis como teflon podem migrar e ser praticamente impossível serem retiradas quando ocorre algum problema ${ }^{6,7,18,27,28}$. Além disso, estas substâncias possuem o inconveniente da imprecisa aferição da quantidade a ser injetada.

A nosso ver, a única desvantagem do uso da fita de PTFE-e está associada ao uso do acesso cervical para a execução da tireoplastia tipo I, pois como to da tireoplastia, o paciente adquire uma cicatriz cervical, o que seria evitado com a técnica por injeção.

Além da complicação de perfuração acidental do seio piriforme durante a manipulação do espaço paraglótico, possível de ocorrer com qualquer substância que se utilize na tireoplastia tipo I para sua colocação, não se detectou qualquer outra complicação. Todos os pacientes estão, até a presente data, com boa e estável qualidade vocal, o que nos encoraja a recomendar o uso deste método no tratamento da PUPV.

\section{CONCLUSÃO}

Pode-se concluir que a fita de PTFE-e demonstrou ser um material bastante satisfatório para a medialização de PV, através da técnica de tireoplastia tipo I, com as vantagens de ser mais fácil e rápida a sua manipulação, permitir um melhor ajuste da PV com a correspondente melhora na qualidade vocal e baixo índice de complicação.

\section{REFERÊNCIASBIBUOGRÁFICAS}

1. Weber RS, Neumayer L, Alford B. Clinical restoration of voice function after loss of the vagus nerve. Head and Neck Surgery 1985; 7:448-57.

2. Hill DP, Meyers AD, Harris J. Autologous fat injection for vocal cord medialization in the canine larynx. Laryngoscope 1991; 101:344-8.

3. Lewy RB. Experience with vocal cord injection. Annals of Otology 1976; 85:440-50.

4. Isshiki NI, Morita H, Okamura H. Thyroplasty as a new phonosurgical technique. Acta Otolaryngology 1974; 78:451-7.

5. Isshiki NI, O kamura H, Ishikawa T. Thyroplasty tipe I for disphonia due to vocal cord paralysis or atrophy. Acta Otolaryngology 1975; 80:465-73.
6. Arnold GE. Alleviation of aphonia or dysphonia through the intracordal injection of teflon paste. Annals of Otology Rhinology \& Laryngology 1963; 72:384-95.

7. Dedo HH, Urrea RD, Lawson L. Intracordal injection of teflon in the treatment of 135 patients with dysphonia. Annals of Otology Rhynology \& Laryngology 1973; 82:661-7.

8. Rubin HJ. Pitfalls in treatment of dysphonias by intracordal injection of synthetics. Laryngoscope 1965; 75:1381-97.

9. Schramm VL, May M, Lavorato AS. Gelfoan paste injection for vocal fold paralysis: temporary rehabilitation of glottic incompetence. Laryngoscope 1978; 88:1258-73.

10. Ford CN, Bless DM. Clinical experience with injectable collagen for vocal fold augmentation. Laryngoscope 1986; 96:863-9.

11. Crumley RL. Update: Ansa cervicalis to recurrent laryngeal nerve anastomosis for unilateral paralysis. Laryngoscope 1991; 101:384288.

12. Mikaelian DO, Lowry LD, Sataloff RT. Lipoinjection for unilateral vocal cord paralysis. Laryngoscope 1991; 101:465-8.

13. Brandenburg JH, Kirkham W, Koschkee D. Vocal cord augmentation with autologous fat. Laryngoscope 1992; 102:495-500.

14. Zaretsky LS, Shindo ML, Rice DH. Autologous fat injection for unilateral vocal fold paralysis. Ann Otol Rhynol Laryngol 1996; 105:602-6.

15. Isshiki N. Progress in laryngeal framework surgery. Acta Otolaryngol 2000; 120:120-7.

16. Kojima H, Hirano S, Isshiki N. Anatomic study for posterior medialization thyroplasty. Ann Otol Rhynol Laryngol 1999; 108:373-7.

17. Isshiki N, Tanabe M, Sawada M. Aryteniod adduction for unilateral vocal cord paralysis. Arch Otolaryngol 1978; 104:555-8.

18. Zeitels SM, Casiano RR, Gardner GM, Hogikyan ND, Koufman JA, Rosen CA. Management of common voice problems: Committee Report. Otolaryngology-Head and Neck Surgery 2002; 126:33348.

19. Giovanni A, Vallicioni J.M, Grass R, Zanaret M. Clinical experience with Gore-Tex for vocal fold medialization. Laryngoscope 1999; 109:284-8

20. McCulloch TM, Hoffman HT, Andrews BT, Karnel MP. Arytenoid adduction combined with Gore-Tex medialization thyroplasty. Laryngoscope 2000; 110:1306-11.

21. Ford CN. Advances and refinements in phonosurgery. Laryngoscope 1999: 109:1891-900.

22. Duke SG, Salomon J, Blalock D, Postma G.N, Koufman JA. Fascia augmentation of the vocal fold: Graft yield in the canine and preliminary clinical experience. Laryngoscope 2001; 111:75964.

23. Brandenburg JH, Unger JM, Koschkee D. Vocal cord injection with autologous fat: A Long-Term Magnetic Ressonance Imaging Evolution. Laryngoscope 1996; 106:174-80.

24. Laccourreye O, Crevier-Buchman L, Le Pimpec-Barthes F, Garcia $D$, Riquet $M$, Brasnu $D$, Recovery of function after intracordal autologous fat injection for unilateral recurrent laryngeal nerve paralysis. The Journal of Laryngology and Otology 1998; 112:10824.

25. Abdel-Aziz MF, Gad El-Hak NA, Carding PN. Thyroplasty for functional rehabilitation of the incompetent larynx. The Journal of Laryngology and 1998; O tology 112:1172-5.

26. Saccogna PW, Werning JW, Setrakian S. Lipoinjection in the paralyzed freline vocal fold: a preliminary clinical study Otolaryngology \& Head \& Neck Surgery 1997; 117:465-70.

27. Kasperbauer JL, Slavit DH, Maragos NE. Teflon granulomas and overinjection of teflon: a therapeutic challenge for the otorhinolaryngologist. Annals of Otology Rhinology \& Laryngology 1993: 102:748-51.

28. Nakayama M, Ford CN, Bless DM. Teflon vocal fold augmentation: failures and management in 28 cases. Otolaryngology \& Head \& Neck Surgery 1993; 109:493-8. 